\title{
Real-time optical differentiation for moire interferometry
}

\author{
Krzysztof Patorski, Daniel Post, Robert Czarnek, and Yifan Guo
}

\begin{abstract}
Real-time methods for differentiation of in-plane displacement fields produced by the moire interferometry technique are introduced. Two approaches are developed: (1) optical shearing of displacement patterns and (2) superposition of two lateral shear interferograms of wavefronts from 2 diffraction orders of the specimen grating. Coherence problems are circumvented by polarization effects. In both cases additive-type moire fringes give the map of displacement derivatives of the object under load. The issue of carrier patterns and extraneous fractional fringe order is clarified. Experimental verification of the principle is presented.
\end{abstract}

\section{Introduction}

The moire interferometry method ${ }^{1-3}$ is a high-sensitivity real-time technique for whole-field mapping of inplane displacements of deformed bodies. A reflectivetype diffraction grating (specimen grating) is fixed to the object under study and illuminated by two mutually coherent collimated beams. First-order diffractions from the specimen grating redirect the two illumination beams into collinear propagation paths. Since the diffracted beams are of opposite signs, they carry mutually conjugate information about the inplane displacements (corresponding to the departure of specimen grating lines from straightness). The wavefront deformations caused by the out-of-plane displacements are the same in both diffraction orders, and their influence is canceled. As a result, the interference pattern in the observation plane provides the map of in-plane displacements with double sensitivity. When using crossed-line specimen gratings and a proper multiple-beam illumination system, the displacement information in two orthogonal directions or three directions can be obtained.

From the point of view of strain analysis, it is not the displacement fields alone that are desired but their partial derivatives. The mechanical shearing technique can be used for this purpose wherein two identical (or positive and negative) copies of sufficiently dense displacement fringe patterns are overlapped and laterally shifted. ${ }^{2}$ The overlapped patterns create moire fringes called moire or moire. They map the derivative of displacement along the shift direction

The authors are with Virginia Polytechnic Institute and State University, Department of Engineering Science \& Mechanics, Blacksburg, Virginia 24061.

Received 29 September 1986.

0003-6935/87/101977-06\$02.00/0.

(C) 1987 Optical Society of America. averaged over the shift distance. This approach was applied to moire interferometry by Weissman and Post. ${ }^{4}$ The same authors in their subsequent work ${ }^{5}$ replaced mechanical shearing with optical shearing. Lateral shear interferograms (with dense tilt fringes) of each diffraction order from the specimen grating were recorded separately and overlapped. Again moire fringes displayed the derivative information.

Although being relatively simple in their implementation, both differentiation methods mentioned possess the common disadvantage of nonreal-time operation. Real-time operation is desired for some mechanical problems, for example, to adjust loading conditions for symmetry. A theoretical proposition for a real-time technique has been suggested by Pirroda. ${ }^{6}$ It is complicated, however, because it requires the use of phase conjugation optics.

The purpose of this work is to describe various optical methods that provide simple real-time differentiation of moire interferometry fields. The methods can be categorized into two main groups called (1) optical shearing of displacement patterns and (2) superimposition of lateral shear interferograms.

\section{Definition of the Problem}

Let us introduce the objective of the studies using Fig. 1. The moire interferometry method employs symmetrical double-beam illumination of the specimen grating (SG) fixed to the object under load. Illuminating beams $A$ and $B$ are mutually coherent and are identically linearly polarized. The vertical polarization direction, parallel to the specimen grating lines, is indicated in Fig. 1. When the incidence angles of $A$ and $B$ are tuned to the first diffraction order angle of $\mathrm{SG}$, the +1 diffraction order of $A$ and the -1 order of $B$ propagate coaxially along the grating normal. The wavefronts of these beams are indicated as $W_{A}$ and $W_{B}$, respectively. The interference pattern is observed at the plane $O P$ conjugate with the specimen grating plane. A single imaging objective $L$ is shown in Fig. 1. However, an afocal imaging system is frequently used instead. 
Now let us introduce a beam-splitting and shearing unit $Q$ into the observation system. At this moment we will not specify its design. Its role is to produce identical replicas $W_{A}^{*}$ and $W_{B}^{*}$ of the wavefronts $W_{A}$ and $W_{B}$, respectively. The beam-splitting and shearing device should create a lateral displacement between $W_{A}$ and $W_{A}^{*}$ and between $W_{B}$ and $W_{B}^{*}$; in certain applications, it will also change the tilt angle between the beams in each pair.

With the unit $Q$ present, four beams, $W_{A}, W_{A}^{*}, W_{B}$, and $W_{B}^{*}$, are encountered simultaneously at the observation plane $O P$. In general, their four-beam interference will lead to interference patterns that are too complicated to analyze. However, if certain beams are made incoherent with respect to the others, information about the derivatives of in-plane displacements can be obtained. Two solutions are available.

(1) Wavefronts in the pairs $W_{A}, W_{B}$ and $W_{A}^{*}, W_{B}^{*}$ are coherent, but one pair is incoherent with respect to the other.

(2) Wavefronts in the pairs $W_{A}, W_{A}^{*}$ and $W_{B}, W_{B}^{*}$ are coherent, but one pair is incoherent with respect to the other.

The first approach is a real-time optical implementation of the mechanical shearing technique. ${ }^{4}$ The second is a real-time version of the optical method described by Weissman et al. ${ }^{5}$

In both cases, carrier fringes can be added when needed to densify the interference patterns. In case (1) the carrier is introduced by detuning the illumination beams $A$ and $B$, and in case (2) the carrier must be generated by a wavefront tilt introduced by element $Q$. Methods of implementation of the two approaches are described.

\section{Optical Shearing of Displacement Patterns}

\section{A. Theoretical Description}

Our objective is to generate two identical in-plane displacement fringe patterns in plane $O P$ and shift them laterally. The interferograms must be mutually incoherent; i.e., two beams forming one interferogram must not interfere with the beams generating the shifted pattern. The incoherence requirement suggests the use of polarized light interferometry techniques.

A proper beam-splitting and shearing device $Q$ can generate the necessary relative displacement of the two-component interferograms. There can be many arrangements serving this purpose. Below we describe only some of them, which according to our opinion are relatively simple to implement. Figure 2 shows the optical system using a single Wollaston prism.

The polarization of symmetrical beams $A$ and $B$ is adjusted to produce $45^{\circ}$ polarization of the diffracted beams (with wavefronts $W_{A}$ and $W_{B}$ ); this condition is symbolized by the superscript 45 on $W_{A}$ and $W_{B}$. As is well known, the Wollaston prism splits each incident beam into two angularly separated beams having mutually perpendicular polarization directions, 0 and $90^{\circ}$ polarizations. At a finite distance from the prism the two beams are laterally separated. These two proper-

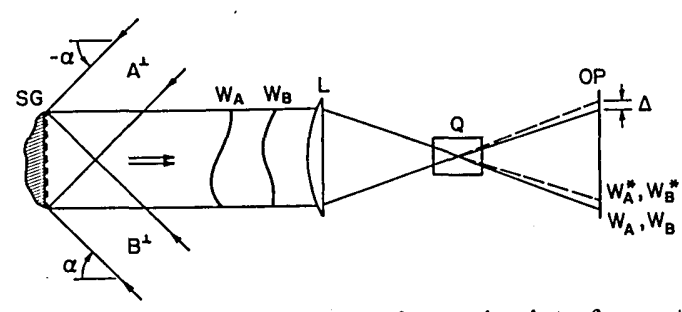

Fig. 1. Schematic representation of a moire interferometry arrangement with an added beam splitting and shearing unit $Q$. SG, specimen grating; $L$, imaging lens; $O P$, observation plane; $W_{A}$ and $W_{B}$, wavefronts of diffraction orders from $S G ; W_{A}^{*}$ and $W_{B}^{*}$, replicas of $W_{A}$ and $W_{B}$, respectively, shifted by the shear amount $\Delta$.

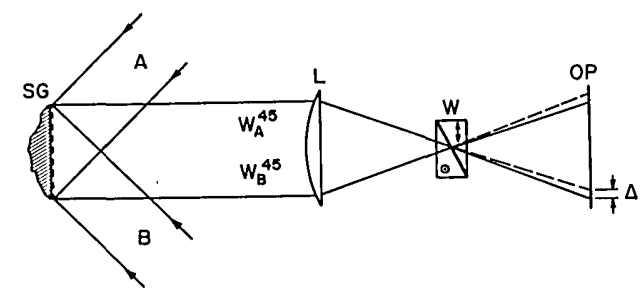

Fig. 2. Real-time arrangement for differentiation of in-plane displacement patterns using the Wallaston prism $W$. Superscript 45 denotes the polarization azimuth.

ties serve our purpose well. Two mutually incoherent interferograms are formed at the observation plane. Each one results from the interference of the same polarization components present in the beams $W_{A}$ and $W_{B}$. The magnitude of the shear can be varied stepwise by using prisms with different splitting angles. To generate derivative fringes with respect to the perpendicular direction, the Wollaston prism can be rotated through $90^{\circ}$ about the center line of the optical system.

A continuous change of the shear magnitude can be realized with the configurations shown in Fig. 3. They use a polarizing beam splitter, which gives a perpendicular separation of beams with orthogonal polarization states. The horizontal polarization components (present in the specimen grating diffraction orders $W_{A}$ and $W_{B}$ ) are transmitted, whereas the vertical ones (parallel to the SG lines) are reflected by the dielectric beam splitting layer. In (a) the quarterwave plates rotate the polarization by $90^{\circ}$ in each arm, with the result that the beams emerge in a direction perpendicular to their entrance. Without the $\lambda / 4$ plates, in (b), they emerge parallel to the direction of entrance and require deflection by a beam splitter. In either case, two interferograms of the in-plane displacement field are produced in $O P$ with 0 and $90^{\circ}$ polarizations, respectively. Generation of the lateral shift between the interferograms in any required direction is easily accomplished by proper tilt of one of the mirrors $M 1$ or $M 2$. The two sheared interferograms add in intensity at the observation plane.

Another version of the arrangement using the polarizing beam splitter is presented schematically in Fig. 4 . By tilting mirror $M$ the shear magnitude can be continuously varied in any desired direction. Additionally, 


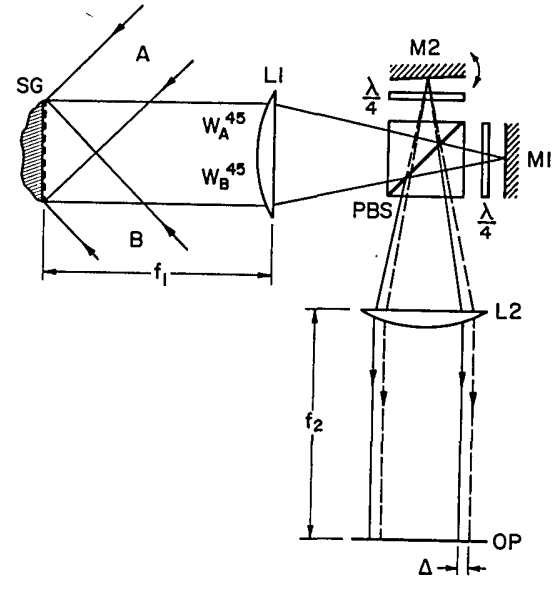

(a)

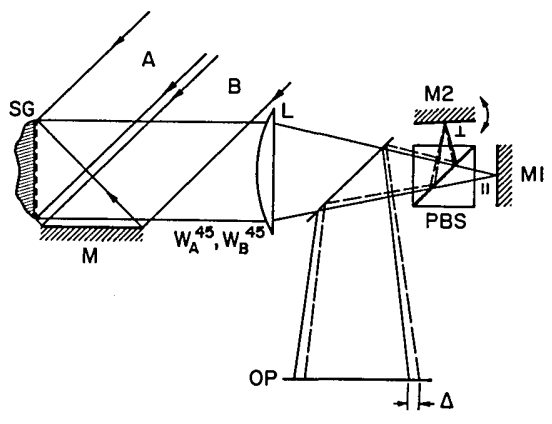

(b)

Fig. 3. (a) Real-time systems using a polarizing beam splitter. Mirror $M$ provides the symmetrical illumination beam. Rotation of one of the mirrors $M 1$ and $M 2$ introduces a lateral shift $\Delta$. (b) Realtime systems using a polarizing beam splitter. Mirror $M$ provides the symmetrical illumination beam. Rotation of one of the mirrors $M 1$ or $M 2$ introduces a lateral shift $\Delta$.

PBS and $M$ can be mounted on a rigid plate and rotated about the optical axis as an alternate means of changing the shear direction.

It is necessary to note that this version of the beam splitting and shearing unit requires the use of an afocal imaging system $L 1-L 2$. This is because the optical path lengths from the specimen grating to the observation plane is different for the two beam pairs forming in-plane displacement interferograms. Therefore, when using a nonafocal imaging system, one of the patterns is out of focus. An afocal imaging configuration eliminates this effect. The configurations of Figs. 2 and 3 can utilize either an afocal or nonafocal imaging system.

If the polarizing beam splitters described above are not available, the system shown in Fig. 5 can be used. This is the arrangement described and analyzed in one of the previous papers ${ }^{5}$ dealing with the double-exposure shearing method. It can be readily adapted for the purpose under consideration by using two linear polarizers $P 1$ and $P 2$ with orthogonal transmission axes. An afocal imaging system $L 1-L 2$ must be used.

With these systems, the derivative map is displayed by means of moire fringes formed by adding two quasiperiodic interferograms. As is well known, the addi-

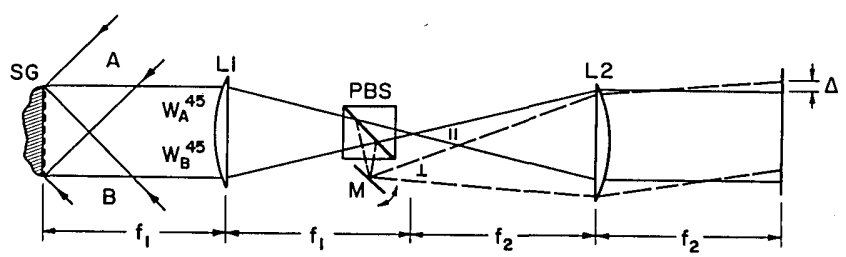

Fig. 4. Optical arrangement using an afocal imaging system $L 1-L 2$ and a polarizing beam splitter.

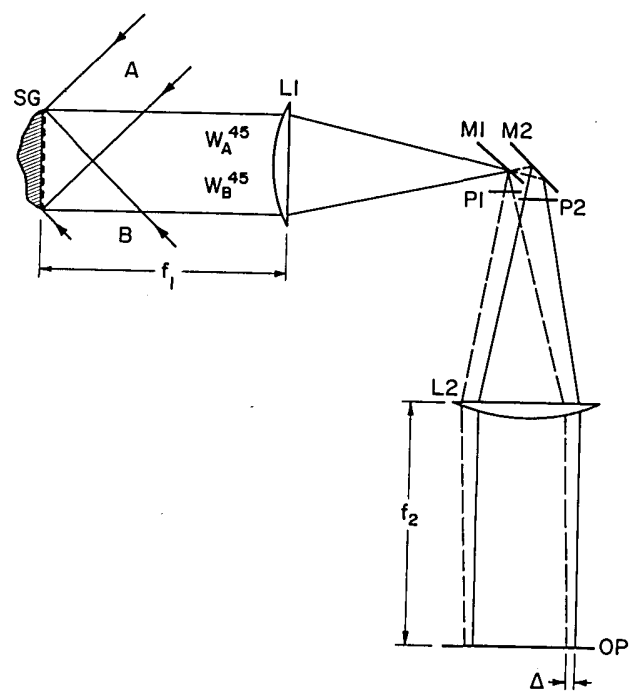

Fig. 5. Analogous optical system that does not require the polarizing beam splitter. $P 1, P 2$, linear polarizers with axes mutually orthogonal.

tion-type moire requires that the individual sheared patterns (here the displacement patterns) must be resolved by the observer. Implications of this are discussed in connection with an experimental demonstration.

\section{B. Experimental Verification}

For experimental verification of the approach of real-time optical shearing of displacement patterns, the arrangement shown in Fig. 3(b) was used. The illumination system utilized a plane mirror $M$ to divide a collimated beam into two symmetrical paths. It is interesting to note that this mirror introduces a phase shift of $\pi$ for light polarized parallel to the grating lines but no phase shift for the orthogonal polarization. Consequently, the interferograms created in 0 and $90^{\circ}$ polarizations are complementary, that is, one is the negative of the other. The same result is achieved with other illumination systems in which an odd number of mirrors is used to form the symmetrical illumination beams.

For this demonstration, the specimen was a cantilever beam, $19 \mathrm{~mm}$ high, $12 \mathrm{~mm}$ thick, and $75 \mathrm{~mm}$ long, made of a stiff epoxy polymer. A crossed-line aluminized specimen grating of 1200 lines/mm was formed on the 19- $\times 75-\mathrm{mm}$ surface by the usual replication technique. ${ }^{1}$ The sensitivity of the resultant displacement field corresponded to that of moire with 2400 lines/mm. 
A small load was applied to the cantilever beam, and mirror $M 2$ was adjusted to produce a shear of $\Delta=1.2$ $\mathrm{mm}$ in the $x$ or lengthwise direction. The result is seen in Fig. 6(a). Note the double image of a cross marked on the specimen, which defines the shear magnitude and direction. Figure 6(b) shows the same conditions, except mirror $M 2$ was adjuted for a shear of $\Delta=0.9 \mathrm{~mm}$ in the $y$ direction.

Figure 7 shows corresponding sheared displacement fields for an increased load and smaller shear distances. The moire fringes of displacement derivatives are well defined in all cases, but different conditions should be explained.

Where the displacement fringes are relatively coarse, the eye can readily distinguish the dark fringes (consisting of black and white stripes where fringes of both displacement fields superimpose in registration) and bright fringes (consisting of a constant brightness where bright fringes of one displacement pattern fall between the bright fringes of the sheared duplicate pattern). The moire fringes can be distinguished as constant brightness zones interposed between striped zones, and for that reason they have real-time visibility to the eye.

In other zones, where the displacement fringes are very closely spaced, the explanation is different. In fact, the dark and bright fringes have equal average intensities. This must be true since the intensity of each of the superimposed patterns is constant when integrated over a region that is large compared to the fringe spacing. Therefore, the integrated intensity must be constant, independent of whether the component fringes interweave or overlap in registration. An observer who cannot resolve the component fringes (the displacement fringes) sees a uniform field devoid of moire fringes.

On a fine or pointwise scale, the intensity within the constant brightness moire fringe is equal to the maximum intensity I of the constituent displacement fringes. Within the striped zone the minimum intensity is zero, and the maximum intensity is $2 I$. A nonlinear observer can distinguish these fringes. For example, if the receiver saturates at intensity level $\mathbf{I}$, it responds equally to $\mathbf{I}$ and $2 \mathbf{I}$. Then the constant brightness fringe retains the brightness corresponding to I, but the striped fringes exhibit (essentially) the average from zero to $I$ or $I / 2$. Where the fringes are too fine to be resolved by the eye, a nonlinear recording medium must be used, and it portrays the moire fringes as white and grey bands. While this is not realtime observation in the true sense, it is real-time recording. This condition prevails in Fig. 7 on the right side. Photographic systems with rapid processing and TV recording systems offer the essential qualities of real-time observation.

The central fringe in Figs. 6(a) and 7(a) along the neutral axis of the beam is a zero-order fringe; the derivative of displacement is zero. It is a constant intensity fringe in this case, because the two sheared displacement patterns are complementary, as noted earlier.

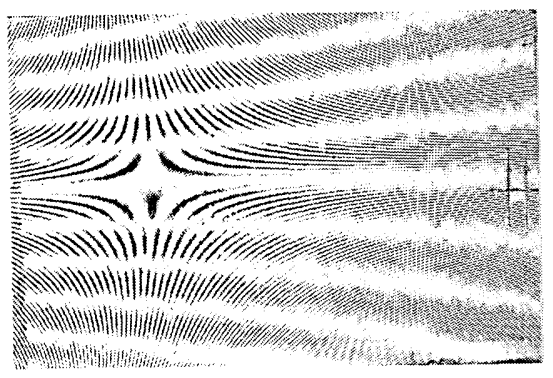

(a)

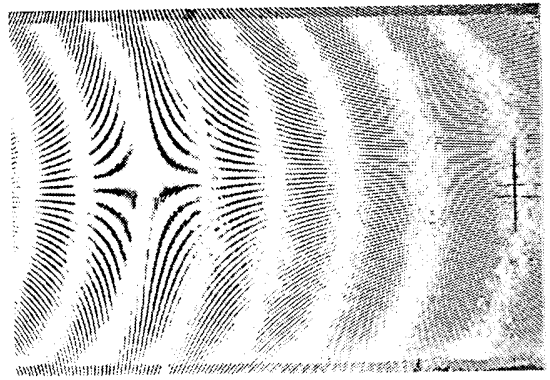

(b)

Fig. 6. Real-time patterns of (a) $\Delta U / \Delta x$ and (b) $\Delta U / \Delta y$ for a cantilever beam. $x$ and $y$ are horizontal and vertical coordinates, respectively. $U$ is the $x$ component of specimen displacements. $x=$ $1.2 \mathrm{~mm} ; y=0.9 \mathrm{~mm}$

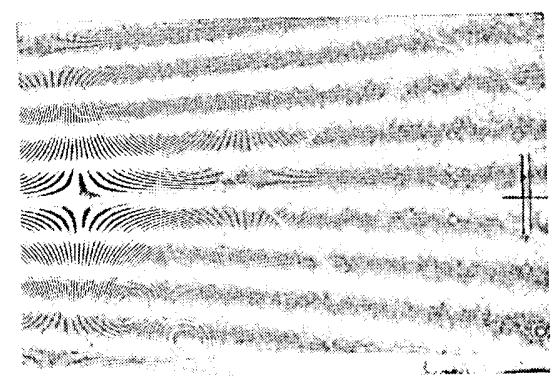

(a)

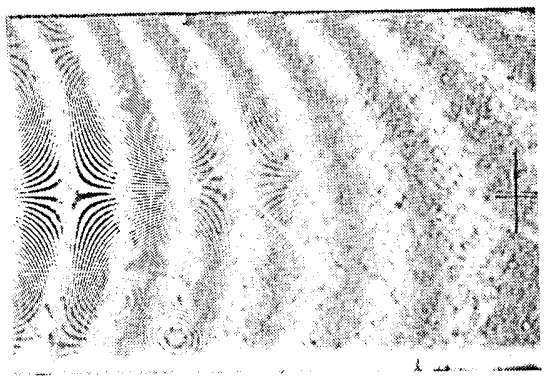

(b)

Fig. 7. Equivalent patterns for increased load level, requiring nonlinear recording. $x=0.5 \mathrm{~mm} ; y=0.3 \mathrm{~mm}$.

\section{Superimposition of Lateral Shear Interferograms}

An interference pattern is formed when beams $W_{A}$ and $W_{A}^{*}$ are sheared; another interference pattern is formed by sheared beams $W_{B}$ and $W_{B}^{*}$. The incoherent superimposition of these two patterns forms a moi$\mathrm{re}$, and it is shown in Ref. 5 that the moire fringes represent derivatives of displacement, just like the first case considered. 
Referring to Fig. 1, the real-time formation of this moire pattern is implemented when wavefronts $W_{A}$ and $W_{B}$ are mutually incoherent. The practical technique is to make the illumination beams $A$ and $B$ mutually incoherent. When $A$ and $B$ are derived from the same source they can be made incoherent by

(1) dissimilar path lengths with the path difference for beams $A$ and $B$ exceeding the coherence length of the laser light source;

(2) dissimilar frequencies of $A$ and $B$, for example, by using acoustooptical methods of frequency shifting;

(3) dissimilar polarizations with polarizations of $A$ and $B$ parallel and perpendicular to the specimen grating lines, respectively.

The desired effect can also be obtained by using two separate laser light sources for $A$ and $B$.

Let us choose for further description the solution with orthogonal polarization states of $W_{A}$ and $W_{B}$. It can be realized by introducing linear polarizers with mutually perpendicular axes into the beams $A$ and $B$. An alternative is to introduce a halfwave plate in one beam. Figure 8 shows, for example, an appropriate modification of the arrangement presented in Fig. 3. Polarization of $A$ and $B$ are vertical and horizontal, respectively. An ordinary nonpolarizing beam splitter is used. The incidence angles of $A$ and $B$ must be adjusted to have $W_{A}$ and $W_{B}$ propagating collinearly. By tilting mirror $M 2$, the lateral shear interferograms of $W_{A}$ and $W_{B}$ appear simultaneously. No carrier fringes are produced with this arrangement when mirrors $M 1$ and $M 2$ are at the back focal plane of lens $L$. Carrier fringes can be introduced to densify the interferograms. In the arrangement of Fig. 8 this can be achieved by axially displacing the beam splitter and mirrors toward lens $L$. Then the beams in both arms of the interferometer are no longer focused at mirrors $M 1$ and M2. If one of them is rotated, the carrier fringes result, since the focal spots of the beams forming each interferogram no longer coincide. They are separated, as seen from the observation plane, along the shear direction. Correspondingly, the carrier fringes run perpendicularly to it. The shear and tilt values are not independent in this configuration; the same remark is valid when the afocal imaging system is used with the shearing arrangement of Fig. 8.

The two lateral shear interferograms are superimposed in registration in the observation plane $O P$. They create a moire pattern, which represents the derivative of displacements of the specimen. The number of fringes in the moire pattern is proportional to the shift $\Delta$. When $\Delta=0$ a uniform intensity distribution is observed, devoid of lateral shear interference fringes and devoid of moire fringes.

Appropriate changes of polarization convert the system of Figs. 4 and 5 for superposition of lateral shear interferograms. In both, the polarization of illumination beams $A$ and $B$ are made parallel and perpendicular to the specimen grating lines; in Fig. 4 a nonpolarizing beam splitter is substituted for PBS; in Fig. 5, polarizers $P 1$ and $P 2$ are removed. These configurations offer the advantage that the shear magnitude and

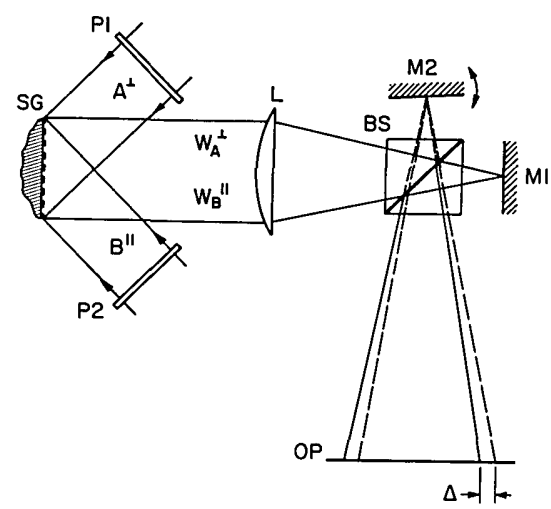

Fig. 8. Real-time arrangement for overlapping the lateral shear interferograms of the specimen grating diffraction orders. $P 1$ and $P 2$ denote linear polarizers with the axes set parallel and perpendicular to the SG lines, respectively.

carrier fringes from the wavefront tilt can be adjusted independently. ${ }^{5}$ The afocal imaging system is required.

In these systems, the polarizing elements introduced in beams $A$ and $B$ must be of high optical quality. Otherwise they introduce extraneous and unequal distortions in wavefronts $W_{A}$ and $W_{B}$, producing extraneous moire fringes in the derivative pattern. As an alternative, polarizing elements of lower optical quality can be positioned in each illumination beam upstream of the beam expanding optics.

\section{Carrier Fringes and the Issue of Fractional Fringe
Orders}

Carrier fringes are commonly used in moire interferometry for various purposes. ${ }^{2}$ One is fringe contrast enhancement offered with carrier patterns and optical filtering. ${ }^{5}$ With the specimen in its initial no-load condition, the properly tuned orientations of beams $A$ and $B$ produce a null field, devoid of displacement fringes. If angle $\alpha$ (Fig. 1) is changed slightly, apparent displacement fringes appear; these are uniformly spaced fringes parallel to the specimen grating lines, and they are called fringes of extension. If instead, the specimen is rotated slightly about the specimen grating normal (or beams $A$ and $B$ are adjusted so that the plane containing their center lines is rotated about the same normal), the apparent displacement fringes are perpendicular to the specimen grating lines; these are called carrier fringes of rotation. When the specimen is loaded, the otherwise regular array of carrier fringes becomes irregular. They carry the information on inplane displacements of the specimen grating.

The carrier fringes discussed here are introduced by beams $A$ and $B$. They should not be confused with the carrier fringes introduced by an element of the shearing system, discussed in Sec. IV.

In the absence of carrier fringes, shearing of the displacement patterns results in moire patterns of well-known interpretation. The zero-order moire fringe (the center of the dark or bright fringe) is a zone of zero derivative of displacement. 
When carrier fringes are present, the quantity being differentiated has two components: (1) the load-induced displacements and (2) the apparent displacements of the carrier pattern. The latter is a uniform fringe gradient; its derivative is zero for a shear direction parallel to the carrier fringes, but it is a nonzero constant for a shear direction perpendicular to the carrier fringes. This constant adds uniformly to the moire fringe orders representing the load-induced derivative.

The constant would usually be an integer plus a fraction. The integer does not alter the moire fringes of displacement derivative, but the fraction moves them by a partial fringe spacing. The zero derivative no longer corresponds to the center of a dark or bright fringe. In practice, a correction can be made if the derivative of displacement is known at some point in the field. Otherwise, the following procedure can be employed. With the desired shear adjustment fixed in the apparatus and with no load on the specimen, tune the carrier pattern to produce a whole-order derivative; this is achieved when the carrier fringes from the two sheared patterns are overlapped accurately in registration (or accurately half a fringe spacing out of registration, as desired). Then when the load is applied, the zero displacement derivatives will correspond to the center of the dark (or bright) moire fringe.

Different conditions exist for the method of Sec. IV: the superimposition of lateral shear interferograms. The method requires that no carrier pattern shall be added to the displacement field. The no-load condition must give a null field of moire interferometry fringes, devoid of fringes. Carrier fringes are introduced in this case by the shearing elements, which introduce identical carrier patterns in the two lateral shear interferograms. However, these are automatically in registration in the observation plane, and consequently their contribution to the moire fringe order is always zero. This appears to be a useful advantage of the method.

\section{Summary}

Optical shearing devices can be built into moire interferometry systems to produce maps of in-plane displacement derivatives. Four beams with displacement information emerge, and their mutual coherence makes the interference pattern unintelligible. In previous solutions, the problem was circumvented by recording the beams in pairs, separately in time, and superimposing these patterns to create a moire that represents the derivative field. In this paper we render the two pairs of beams mutually incoherent to achieve the same result but in real time.

Two classes of solution are described in which

(1) the displacement field generated by moire interferometry is sheared to introduce a shifted incoherent duplicate field, and

(2) the two wavefronts that would otherwise produce a moire interferometry pattern are individually sheared; two wavefront sheared interferograms are produced and superimposed in registration.
The required incoherence is introduced by orthogonal polarizations in both cases.

The displacement fields are generated as additive moire fringes (fringes of the sum of two quasi-periodic intensity distributions). As such, the component patterns must be fully resolved in the observation plane. Where the component patterns are coarse, the moire fringes are readily observed by eye in real time. When the component patterns are fine, a nonlinear recording medium is required, and real-time recording is achieved.

An experimental demonstration is provided for one implementation. The high sensitivity of moire interferometry and easy adjustment of the shear magnitude allow use of small shear distances.

Carrier patterns developed in the basic moire interferometry system can be used with solution (1). When the shearing direction is perpendicular to the carrier fringes, a fractional fringe order might be added to the moire fringes; a technique is suggested to eliminate the fractional fringe order. For solution (2), carrier fringes are introduced by the shearing device, and the moire interferometry system should be tuned for zero carrier fringes; no fractional fringe discrepancies are encountered, and no compensatory adjustments are needed.

All the suggested optical systems for producing maps of displacement derivatives can also produce displacement maps. A minor alteration of polarization, obstruction of one element of the shearing device, or both will convert the apparatus to a normal moire interferometry configuration.

The authors gratefully acknowledge the support of the Office of Naval Research under research grant N00014-86-K-0255. The kind assistance of faculty and staff of the Engineering Science and Mechanics Department, VPI and SU, is greatly appreciated. K. Patorski gratefully acknowledges the Warsaw Technical University for granting his leave to VPI and SU.

\section{References}

1. D. Post, "Developments in Moire Interferometry," Opt. Eng. 21, 458 (May 1982).

2. D. Post, "Moire Interferometry," in SEM Handbook on Experimental Mechanics, A. S. Kobayashi, Ed. (Prentice-Hall, Englewood Cliffs, NJ, 1986).

3. A. McDonach, J. McKelvie, P. Mackenzie, and C. A. Walker, "Improved Moire Interferometry and Applications in Fracture Mechanics, Residual Stress and Damaged Composites," Exp. Tech. 23, No. 2, 20 (June 1983).

4. E. M. Weissman and D. Post, "Full-Field Displacement and Strain Rosettes by Moire Interferometry," Exp. Mech. 22, No. 9 , 324 (1982).

5. E. M. Weissman, D. Post, and A. Asundi, "Whole-Field Strain Determination by Moire Shearing Interferometry," J. Strain Anal. 19, No. 2, 77 (1984).

6. L. Pirroda, "Strain Analysis by Grating Interferometry," Opt. Laser Eng. 5, No. 1, 7 (1984). 